\title{
Odor Identification Impairment and Change with Cholinesterase Inhibitor Treatment in Mild Cognitive Impairment
}

\author{
D. P. Devanand ${ }^{\mathrm{a}, \mathrm{b}, *}$, Xinhua Liu $^{\mathrm{c}}$, Richard E. Chunga ${ }^{\mathrm{a}}$, Hannah Cohen $^{\mathrm{a}}$, Howard Andrews ${ }^{\mathrm{c}, \mathrm{e}}$, \\ Peter W. Schofield ${ }^{\mathrm{f}, \mathrm{g}}$, Yaakov Stern ${ }^{\mathrm{b}}$, Edward D. Huey ${ }^{\mathrm{b}}$, Jongwoo Choi ${ }^{\mathrm{d}, \mathrm{e}}$ and Gregory H. Pelton ${ }^{\mathrm{a}}$ \\ ${ }^{a}$ The Memory Disorders Center and the Division of Geriatric Psychiatry at the New York State Psychiatric \\ Institute, New York, NY, USA \\ ${ }^{\mathrm{b}}$ Department of Neurology and the Taub Institute for Research in Alzheimer's disease at Columbia University \\ Medical Center, New York, NY, USA \\ ${ }^{\mathrm{c}}$ The Mailman School of Public Health at Columbia University Medical Center, New York, NY, USA \\ ${ }^{\mathrm{d}}$ Mental Health Data Science at the New York State Psychiatric Institute, New York, NY, USA \\ ${ }^{\mathrm{e}}$ Department of Biostatistics, Columbia University, New York, NY, USA \\ ${ }^{\mathrm{f}}$ The University of Newcastle, Newcastle, Australia \\ $\mathrm{g}^{\mathrm{g}}$ Hunter New England Local Health District, New Lambton, Australia
}

Handling Associate Editor: David Loewenstein

Accepted 18 March 2020

\begin{abstract}
.
Background: Anticholinergic challenge can induce odor identification impairment that indicates Alzheimer's disease (AD) pathology, and short-term change in odor identification impairment with cholinesterase inhibitor (CheI) treatment may predict longer term cognitive outcomes.

Objective: In patients with mild cognitive impairment (MCI) treated prospectively with donepezil, a CheI, for 52 weeks, to determine if 1) acute decline in odor identification ability with anticholinergic challenge can predict cognitive improvement, and 2) change in odor identification over 8 weeks can predict cognitive improvement.

Methods: MCI was diagnosed clinically without AD biomarkers. At baseline, the University of Pennsylvania Smell identification Test (UPSIT) was administered before and after an anticholinergic atropine nasal spray challenge. Donepezil was started at $5 \mathrm{mg}$ daily, increased to $10 \mathrm{mg}$ daily if tolerated, and this dose was maintained for 52 weeks. Main outcomes were ADAS-Cog total score and Selective Reminding Test (SRT) total immediate recall score measured at baseline, 26 and 52 weeks.

Results: In 100 study participants, mean age 70.14 (SD 9.35) years, atropine-induced decrease in UPSIT score at baseline was not associated with change in ADAS-Cog or SRT scores over 52 weeks. Change in UPSIT score from 0 to 8 weeks did not show a significant association with change in the ADAS-Cog or SRT measures over 52 weeks.
\end{abstract}

\footnotetext{
*Correspondence to: D.P. Devanand, MD, New York State Psychiatric Institute, 1051 Riverside Drive, Unit 126, New York, NY 10032, USA. Tel.: +1 646774 8658; Fax: +1 646774 6398; E-mail: dpd3@cumc.columbia.edu.
} 
Conclusion: These negative findings in a relatively large sample of patients with MCI did not replicate results in much smaller samples. Change in odor identification with anticholinergic challenge, and over 8 weeks, may not be useful predictors of cognitive improvement with CheI in patients with MCI.

Keywords: Acetylcholine, Alzheimer's disease, atropine, mild cognitive impairment, olfaction

\section{INTRODUCTION}

In the early pathological stages of Alzheimer's disease $(\mathrm{AD})$, neurofibrillary tangles develop in the olfactory bulb and extend to olfactory projection areas in limbic regions, including the piriform cortex, amygdala, entorhinal cortex, hippocampus, and orbitofrontal cortex [1-5]. Clinically, this manifests as impairment in performance on tests of olfaction, particularly standardized odor identification tests $[6$, 7]. Odor identification impairment demonstrates predictive utility that may be superior to episodic verbal memory impairment for the transition from cognitively normal to mild cognitive impairment (MCI), and shows comparable predictive accuracy for the transition from MCI to AD [6, 8-10].

Acetylcholine was the first major neurotransmitter identified as being deficient in AD [11]. Subsequently, cholinesterase inhibitors (CheI) were developed as medications to treat AD. Dopaminergic, noradrenergic, serotonergic, and particularly cholinergic pathways are involved in olfactory sensory processing [12]. Neurons with choline acetyltransferase are present in all olfactory bulb layers [13, 14] and dendrites of olfactory periglomerular or granule cells show prominent cholinergic synapses [13]. In mice, olfactory bulbectomy leads to degeneration of medial septal cholinergic neurons, and treatment with donepezil, a CheI, is neuroprotective against this effect [15]. In the olfactory bulb, cholinergic receptors are mainly muscarinic [16].

There is some evidence in small samples that odor identification impairment may help to identify which patients with MCI will benefit from CheI treatment. In a small study of 25 patients, donepezil treatment was associated with improvement in odor identification and global functioning [17]. In a double-blind, placebo-controlled trial of 18 patients with depression and MCI, impaired odor identification at baseline was associated with improved episodic verbal memory after donepezil treatment [18]. In another report, greater worsening in odor identification test performance following an intranasal challenge with atropine, an anticholinergic medication, correlated with left hippocampal volume and was associated with the presence of the apolipoprotein $\mathrm{E} \varepsilon 4$ allele [19].

In our pilot study of 37 patients with amnestic MCI who received donepezil for a year, atropine nasal spray challenge at baseline induced a shortterm odor identification decline on the University of Pennsylvania Smell Identification Test (UPSIT) that was associated with weakly significant longer term improvement in episodic verbal memory but not in other measures [20]. In that sample, improvement in odor identification over 8 weeks tended to predict longer term cognitive improvement [20].

In order to establish impaired odor identification in response to anticholinergic challenge as a method to select patients with MCI for CheI treatment, we aimed to replicate these findings in a larger, independent sample with no patient overlap with the prior study. If the findings were replicated, the results would help to identify which patients with MCI are likely to improve with cholinesterase inhibitors, and to avoid prescribing them in patients who are not likely to improve with such treatment.

\section{METHODS}

\section{Participants}

This study was approved by the New York State Psychiatric Institute (NYSPI)/Columbia University Institutional Review Board (IRB). Participants were recruited from the Memory Disorders Clinic at NYSPI and the Behavioral Neurology practice at Columbia University Medical Center (CUMC), and by advertising in local media. Inclusion/Exclusion criteria were essentially the same as in the published pilot study [20]. Inclusion criteria were age 55-95 years and diagnosis of amnestic MCI based on the presence of subjective memory complaints, score $>1.5$ SD below standardized norms on the Wechsler Memory Scale-III Logical Memory combined Story $\mathrm{A}+\mathrm{B}$ immediate recall or delayed recall scores, or the Free and Cued Selective Reminding Test (FC-SRT) immediate or delayed recall score [21], and without functional impairment consistent with dementia. Patients with amnestic MCI, either solely amnestic or 
amnestic with additional cognitive domain deficits, were included. Other inclusion criteria were Folstein Mini-Mental State Exam (MMSE) $\geq 23 / 30$ [22], Clinical Dementia Rating (CDR) score of 0.5 [23], availability of an informant, and ability to provide consent.

Exclusion criteria included current use of cholinesterase inhibitors, history of intolerance or contraindication to donepezil, and use of medications with anticholinergic properties including diphenhydramine, tricyclic antidepressants, and antipsychotics. Benzodiazepine use in lorazepam dose equivalents less than $2 \mathrm{mg}$ daily was permitted in order to enhance generalizability because these medications are commonly prescribed in older adults. Other exclusion criteria were severe unstable medical illness, specific neurological disorders including Parkinson's disease, multiple sclerosis, stroke with residual neurological deficits, psychotic disorders including schizophrenia, bipolar disorder and schizoaffective disorder, alcohol/substance dependence in the past 6 months, current major depression, and suicidality. Exclusion criteria for olfaction testing were current smoker $>1$ pack daily, current upper respiratory infection, nasal trauma or sinus surgery, and head trauma with loss of consciousness.

The screening visit comprised a medical, psychiatric, and neurological evaluation, cognitive assessment to determine inclusion criteria, and blood was drawn for hematocrit, electrolytes, liver, kidney, and thyroid function tests, folate, vitamin B12 levels, and urinalysis to exclude primary medical causes of cognitive impairment.

The subsequent baseline (week 0) visit involved assessment with the two main cognitive outcome measures: ADAS-Cog (11-item version) [24] and 12item 6-trial Selective Reminding Test (SRT) [25]. For diagnostic purposes, neuropsychological tests in the National Alzheimer's Coordinating Center-Uniform Data Set (NACC-UDS) battery were administered: WMS-III digit span forward and backward, WAIS-R digit symbol, Trail Making A and B, verbal fluency for controlled word association using the letters $\mathrm{C}$, $\mathrm{F}$, and $\mathrm{L}$ and animal, vegetable and fruit list generation, Boston Naming Test (60 items). If a participant's preferred language was Spanish, neuropsychological tests were administered in Spanish using standardized versions that have been validated in other studies [6]. Otherwise, tests were administered in English to patients, including bilingual patients who were fluent in English and preferred to be tested in English.
The study physician completed the NACC clinical assessment, Clinician's Interview Based Impression of Change-plus (CIBIC-plus) and Clinician Interview Based Impression of Severity (CIBIS) global assessment ratings, CDR, and the Treatment Emergent Symptoms Scale (TESS) that evaluates 26 common somatic side effects that include gastrointestinal and central nervous system (CNS) side effects known to occur with CheI. An informant completed the Pfeffer Functional Activities Questionnaire (FAQ). Apolipoprotein E genotyping was conducted at Lgc Genomics, a reference laboratory.

\section{Materials}

The University of Pennsylvania Smell Identification test (UPSIT) was administered. This standardized scratch and sniff test consists of 40 booklet pages with a single odor embedded in a microcapsule on each page. Scratching with a pencil releases the odor and the participant checks one of 4 choices, e.g., chocolate, banana, onion, fruit punch. The total UPSIT score ranges from 0 (all answers incorrect) to 40 (all answers correct). At the baseline visit, immediately after UPSIT administration, atropine $1 \mathrm{mg}$, with the dose divided approximately equally between the two nostrils, was administered using the "squirt system" [26]. The patient then assumed the "Mecca" position for 2 min to ensure that the atropine crossed the cribriform plate into the olfactory bulb [19]. The UPSIT was repeated $45 \mathrm{~min}$ later to ensure sufficient time for the atropine to take effect.

\section{Treatment}

Research assessments were repeated at 8,26 , and 52 weeks with the exception of the diagnostic neuropsychological battery that was repeated only at 26 and 52 weeks in order to reduce practice effects. At baseline, donepezil was started at $5 \mathrm{mg}$ daily followed by assessment at 4 weeks for tolerability before increasing the dose to $10 \mathrm{mg}$ daily. This dose was kept constant for the rest of the 52-week study. Patients who could not tolerate donepezil $10 \mathrm{mg}$ were maintained at $5 \mathrm{mg}$; if the $5 \mathrm{mg}$ dose was not tolerated the patient was switched to galantamine or rivastigmine. Twelve patients received galantamine $(n=9)$ or rivastigmine $(n=3)$ during the trial; their data were combined with donepezil data for analysis. Two experienced raters (Drs. Devanand and Stern) made a consensus diagnosis at baseline, 26 and 52 weeks 
while remaining blind to scores on predictor (UPSIT) and cognitive outcome (ADAS-Cog total score and SRT total immediate recall) measures. The study physician completing the CIBIC-plus, CIBIS, and FAQ also remained blind to the cognitive outcome measures.

The main hypotheses were that the acute decrease in UPSIT scores from pre- to post-atropine nasal spray challenge, which indicates greater cholinergic deficiency, would be associated with cognitive improvement on donepezil from baseline to 26 weeks and 52 weeks, and that the increase in UPSIT scores from baseline to 8 weeks (improved olfaction ability on donepezil) would be associated with cognitive improvement from baseline to 26 and 52 weeks. Conversion to the clinical diagnosis of dementia based on consensus diagnosis including CDR, as well as changes in the CIBIC-plus, CIBIS, and FAQ, were examined in secondary analyses.

\section{Statistical analyses}

Variables measured at baseline were described using means and standard deviations for continuous measures and percentages for categorical measures. Spearman correlation coefficient was calculated for bivariate associations between baseline quantitative variables. Linear models with repeated measures were applied to the cognitive outcomes, where the outcomes with right-skewed distributions were properly transformed to reduce impact of extreme values. In particular, logarithmic transformation was used for outcomes with positive values and square root transformation for outcomes with non-negative values. To examine whether dropout was at random, i.e., dependent on any baseline variable, Fisher's exact test and Wilcoxon rank sum test were used to detected differences in categorical and continuous baseline variables between those who completed follow-up and those who dropped out. The factors individually related to dropout were included in a logistic model for dropout to identify the baseline factors predicting dropout and the information was used for data weighting. To estimate parameters in the linear models with repeated measures, the weighted generalized estimating equation approach, using all available data and robust to misspecification of correlation structure in repeated measures, was used to account for dropout at random. For each outcome variable, patients with data missing at baseline were excluded from analysis, while the few intermittent missing data at week $26(n=2$ for ADAS-Cog and SRT total immediate recall) were imputed based on the regression model for the week 26 outcome with the predictors of week 8 measures and relevant baseline variables.

Several statistical models were evaluated. To examine the outcome of change from baseline to week 26 and to week 52, the initial model had the categorical predictor of time or dummy variables indicating specific weeks, without other variables. The second model added baseline variables of age, sex, education, UPSIT0 (UPSIT score at pre-atropine challenge), apolipoprotein E $\varepsilon 4$, and smoking history as covariates, and predictors of UPSIT response to atropine challenge ( $\triangle$ UPSIT), defined as post-pre atropine challenge UPSIT score difference, and its interaction with time variables. The coefficients of the interaction terms indicated whether the time trend in outcome was modified by $\triangle$ UPSIT, or if there was time-varying association between $\triangle$ UPSIT and the outcome. The variable for apolipoprotein $\mathrm{E} \varepsilon 4$ status had categories of positive, negative, and unknown, and the variable for smoking history had categories of non-smoker, past smoker (not smoking in past month), current smoker (smoking in the past month) and unknown status. Variables for apolipoprotein E $\varepsilon 4$ status and smoking history were kept in the final models if they were still related to the cognitive outcomes. The final model also excluded non-significant interaction terms. Similar analyses were conducted for the change in UPSIT score from 0 to 8 weeks as a predictor of the same cognitive outcomes measured over 52 weeks.

Statistical tests were two-sided and significance level was set at 0.05 . All statistical analyses were conducted using SAS version 9.4 (SAS Institute, Cary, NC).

\section{RESULTS}

Table 1 describes the baseline demographic and clinical characteristics of the sample. Of 131 participants excluded for not meeting inclusion criteria, 102 did not meet criteria for amnestic MCI. In the sample of 100 participants, 61 participants met criteria for single domain amnestic MCI, and 8 patients were tested in Spanish. Two patients were taking benzodiazepines (Clonazepam $0.5 \mathrm{mg}$ and $1.0 \mathrm{mg}$ daily, respectively). Fifty-one patients reported a history of smoking and 14 of them were current smokers. Mean baseline UPSIT (pre-atropine challenge) score was 26.0 (SD 7.00) and did not differ significantly between males and females. As expected, higher 
Table 1

Baseline characteristics of 100 participants with mild cognitive impairment

\begin{tabular}{|c|c|c|c|}
\hline & $\mathrm{N}$ & $\%(n)$ & \\
\hline Sex & 100 & & \\
\hline Male & & $49.00(49)$ & \\
\hline Female & & $51.00(51)$ & \\
\hline Race/Ethnicity & 100 & & \\
\hline White (Non-Hispanic) & & $55.00(55)$ & \\
\hline African-American & & $22.00(22)$ & \\
\hline Hispanic & & $20.00(20)$ & \\
\hline Asian & & $3.00(3)$ & \\
\hline Apolipoprotein & 100 & & \\
\hline$\varepsilon 4+$ & & $28.00(28)$ & \\
\hline$\varepsilon 4-$ & & $65.00(65)$ & \\
\hline Unknown & & $7.00(7)$ & \\
\hline Smoking status & 100 & & \\
\hline Never & & $45.00(45)$ & \\
\hline Past & & $34.00(34)$ & \\
\hline Current & & $13.00(13)$ & \\
\hline \multirow[t]{2}{*}{ Unknown } & & $8.00(8)$ & \\
\hline & & Mean \pm SD & Range \\
\hline$\overline{\text { Age }}$ & 100 & $70.14 \pm 9.35$ & 55,89 \\
\hline Years of schooling & 100 & $15.83 \pm 3.38$ & 7,23 \\
\hline MMSE & 100 & $26.72 \pm 2.17$ & 21,30 \\
\hline UPSIT score at baseline & 100 & $26.00 \pm 7.75$ & 10,38 \\
\hline Post-pre nasal challenge & 99 & & \\
\hline UPSIT score change & & $-0.92 \pm 3.77 *$ & $-19,9$ \\
\hline UPSIT reduction $>25 \%$ & & $7.07(7)$ & \\
\hline Over first 8 weeks & 90 & & \\
\hline UPSIT score change & & $0.49(4.40)$ & $-12,11$ \\
\hline UPSIT reduction $>25 \%$ & & $5.56 \%(5)$ & \\
\hline ADAS-Cog & 99 & $11.41 \pm 4.67$ & $3,25.67$ \\
\hline SRT-total recall & 100 & $36.14 \pm 10.67$ & 15,62 \\
\hline SRT-delayed & 100 & $4.07 \pm 2.63$ & 0,11 \\
\hline WAIS-R digit symbol & 99 & $36.83 \pm 13.72$ & 9,68 \\
\hline FAQ & 94 & $4.80 \pm 4.84$ & 0,24 \\
\hline ECog & 92 & $67.93 \pm 20.58$ & 39,130 \\
\hline CIBIS & 98 & $3.05 \pm 0.58$ & 2,4 \\
\hline TESS & 98 & $2.57 \pm 2.62$ & 0,11 \\
\hline
\end{tabular}

* $p=0.0172$ from paired $T$-test to detect UPSIT score change. MMSE, Mini-Mental Status Exam (Folstein); ADAS-Cog, Alzheimer's Disease Assessment Scale-Cognitive (11 items, higher scores indicate worse cognitive performance); SRT, Selective Reminding Test 12-item 6-trial version (higher scores indicate better cognitive performance); UPSIT, University of Pennsylvania Smell Identification test (40 items, range 0-40); WAIS-R digit symbol, Wechsler Adult Intelligence Test-Revised Digit Symbol; FAQ, Pfeffer Functional Activity Questionnaire; ECog, Everyday Cognition; CIBIS, Clinician's Interview Based Impression of Severity; TESS, Treatment Emergent Symptom Scale.

UPSIT scores were associated with higher MMSE (Spearman correlation coefficient $\mathrm{r}=0.37, p<0.001$ ) and SRT total immediate recall $(\mathrm{r}=0.31, p<0.01)$ scores, and with lower age $(\mathrm{r}=-0.36, p<0.0001)$ and lower ADAS-Cog scores $(\mathrm{r}=-0.32, p<0.01)$ at baseline. Of 100 recruited participants, 23 dropped out and 77 completed the 52-week study. Patients who dropped out were less likely to be Apolipoprotein $\mathrm{E} \varepsilon 4$ positive, and were older with higher
ADAS-Cog score (worse cognition) and lower SRT score (worse cognition) at baseline (Supplementary Table 1). Logistic regression analysis, however, suggested that when baseline age was included in the model for dropout, other factors were no longer associated with dropout.

Cognitive performance improved over 52 weeks, with a decrease in ADAS-Cog scores and an increase in SRT total recall scores ( $\mathrm{ps}<0.05$ ). Table 2 shows that ADAS-Cog score changed from mean 11.41 (SD 4.67) at baseline to 10.13 (SD 4.22) at 8 weeks, 9.63 (SD 4.44) at 26 weeks, and 10.40 (SD 4.72) at 52 weeks (Table 2). SRT total immediate recall score changed from 36.14 (SD 10.67) at baseline to 39.99 (SD 10.30) at 8 weeks, 39.75 (SD 10.69) at 26 weeks, and 39.62 (SD 11.44) at 52 weeks. Baseline UPSIT score was not associated with changes in either ADAS-Cog or SRT total recall scores over the 52-week study.

All $100 \mathrm{MCI}$ participants had CDR $=0.5$ at baseline. One of the 5 participants who was rated as $\mathrm{CDR}=1$ at 52 weeks (mild dementia) also had $\mathrm{CDR}=1$ at 26 weeks. One participant improved to $\mathrm{CDR}=0$ at 26 weeks and 9 participants had $\mathrm{CDR}=0$ at 52 weeks. The majority of participants remained at $\mathrm{CDR}=0.5$ at 26 weeks $(n=75)$ and 52 weeks $(n=62)$. The ten patients with improved CDR were younger (mean age 66 years) and tended to have better performance on cognitive tests at baseline than those who did not have CDR improvement (mean age 70.6 years), but the differences were not statistically significant.

\section{Acute decrease in UPSIT scores with atropine challenge}

From pre- to post-atropine challenge, as expected, UPSIT scores decreased an average of 0.92 (SD $3.77)$ points $(p=0.017)$. The acute change in UPSIT scores with atropine challenge was correlated with baseline UPSIT scores $(\mathrm{r}=-0.23, n=99$, $p<0.05$ ) while unrelated to past or current smoking status.

Among patients who completed the week 52 visit, two patients had missing ADAS-Cog data at week 26. Imputation for these intermittent missing data was based on a model from available data $(n=81$ patients) with good fitting $\left(\mathrm{R}^{2}=0.5823\right)$ : ADAS-Cog at 26 weeks $=\exp (0.53278+0.32437 \log ($ baseline ADAS-Cog) + $0.46067 \log ($ ADAS-Cog at weeks 8) 0.26988 male). The initial linear model with repeated measures fit to 264 observations from 99 patients, 
Table 2

Cognitive measures, UPSIT scores, and clinical variables by time-point

\begin{tabular}{|c|c|c|c|c|}
\hline & Baseline & Week 8 & Week 26 & Week 52 \\
\hline & Mean \pm SD (n) & Mean \pm SD (n) & Mean \pm SD (n) & Mean \pm SD (n) \\
\hline UPSIT & $26 \pm 7.75(100)$ & $26.81 \pm 7.83(90)$ & $26.73 \pm 7.38(83)$ & $26.67 \pm 6.94(76)$ \\
\hline \multicolumn{5}{|l|}{ Primary outcomes } \\
\hline ADAS-Cog & $11.41 \pm 4.67(99)$ & $10.13 \pm 4.22(89)$ & $9.63 \pm 4.44(83)$ & $10.40 \pm 4.72(77)$ \\
\hline SRT-Total & $36.14 \pm 10.67(100)$ & $39.99 \pm 10.30(90)$ & $39.75 \pm 10.69(83)$ & $39.62 \pm 11.44(77)$ \\
\hline CIBIC-plus & - & $3.34 \pm 0.74(89)$ & $3.15 \pm 0.86(82)$ & $3.11 \pm 1.17(76)$ \\
\hline \multicolumn{5}{|l|}{ Secondary outcomes } \\
\hline SRT-delayed & $4.07 \pm 2.63(100)$ & $4.32 \pm 2.95(90)$ & $4.58 \pm 3.12(83)$ & $4.19 \pm 3.07(77)$ \\
\hline WAIS-R digit symbol & $36.83 \pm 13.72(99)$ & - & - & $37.24 \pm 12.17(76)$ \\
\hline FAQ10 & $4.80 \pm 4.84(94)$ & $4.68 \pm 4.56(83)$ & $4.84 \pm 4.96(77)$ & $4.25 \pm 4.76(74)$ \\
\hline ECOG & $67.93 \pm 20.58(92)$ & $67.88 \pm 23.35(83)$ & $67.92 \pm 23.20(77)$ & $66.84 \pm 22.66(74)$ \\
\hline CIBIS & $3.05 \pm 0.58(98)$ & $2.83 \pm 0.57(89)$ & $2.65 \pm 0.62(82)$ & $2.70 \pm 0.71(76)$ \\
\hline \multicolumn{5}{|l|}{ Clinical variable } \\
\hline TESS & $2.57 \pm 2.62(98)$ & $1.61 \pm 1.90(88)$ & $1.42 \pm 1.86(77)$ & $1.28 \pm 2.11(74)$ \\
\hline MMSE & $26.72 \pm 2.17(100)$ & - & $26.90 \pm 2.20(83)$ & $26.44 \pm 2.47(77)$ \\
\hline CDR & $0.5 \pm 0(99)$ & - & $0.48 \pm 0.12(80)$ & $0.47 \pm 0.21(76)$ \\
\hline
\end{tabular}

ADAS-Cog, Alzheimer's Disease Assessment Scale-Cognitive (11 items, higher scores indicate worse cognitive performance); SRT, Selective Reminding Test 12-item 6-trial version (higher scores indicate better cognitive performance); UPSIT, University of Pennsylvania Smell Identification test (40 items, range 0-40); CIBIC-plus, Clinician's Interview Based Impression of Change-plus; WAIS-R digit symbol, Wechsler Adult Intelligence Test-Revised Digit Symbol; FAQ, Pfeffer Functional Activity Questionnaire; Ecog, Everyday Cognition; CIBIS, Clinician's Interview Based Impression of Severity; TESS, Treatment Emergent Symptom Scale; MMSE, Mini-Mental Status Exam (Folstein); CDR, Clinical Dementia Rating Scale.

Table 3

Linear models with repeated measures of cognitive outcomes over time

\begin{tabular}{|c|c|c|c|c|}
\hline & Initial model & Final mode & & \\
\hline Outcome: $\log$ (ADAS-cog) & $\mathrm{B}(95 \% \mathrm{CI})$ & $\mathrm{B}(95 \% \mathrm{CI})$ & TS (df) & $P$ \\
\hline Week 26 versus 0 & $-0.172(-0.264,-0.081)$ & $-0.134(-0.221,-0.048)$ & $9.46(2)$ & 0.0088 \\
\hline Week 52 versus 0 & $-0.096(-0.190,-0.001)$ & $-0.067(-0.157,0.023)$ & & \\
\hline Male versus female & & $-0.116(-0.250,0.018)$ & $2.89(1)$ & 0.089 \\
\hline Education & & $-0.017(-0.036,0.002)$ & $3.20(1)$ & 0.074 \\
\hline Baseline Age & & $0.016(0.009,0.023)$ & $20.16(1)$ & $<0.0001$ \\
\hline ApoE $\varepsilon 4$ positive versus negative & & $0.210(0.068,0.352)$ & $8.35(1)$ & 0.038 \\
\hline ApoE $\varepsilon 4$ unknown versus negative & & $-0.002(-0.166,0.162)$ & $0.0004(1)$ & 0.98 \\
\hline Baseline UPSIT & & $-0.011(-0.020,-0.002)$ & $5.43(1)$ & 0.020 \\
\hline$\triangle$ UPSIT & & $-0.039(-0.059,-0.018)$ & $13.91(1)$ & 0.0002 \\
\hline$\triangle$ UPSIT by week 26 & & $0.031(0.003,0.060)$ & $4.76(2)$ & 0.093 \\
\hline$\Delta$ UPSIT by week 52 & & $0.021(-0.009,0.052)$ & & \\
\hline \multicolumn{5}{|l|}{ Outcome: SRT-total recall } \\
\hline Week 26 versus 0 & $2.704(0.954,4.454)$ & $3.265(1.060,5.471)$ & $15.54(2)$ & 0.0004 \\
\hline Week 52 versus 0 & $2.661(0.906,4.416)$ & $4.105(1.990,6.221)$ & & \\
\hline Male versus female & & $2.543(-0.702,5.788)$ & $2.37(1)$ & 0.12 \\
\hline Education & & $0.300(-0.201,0.801)$ & $1.37(1)$ & 0.24 \\
\hline Baseline Age & & $-0.597(-0.770,-0.424)$ & $46.10(1)$ & $<0.0001$ \\
\hline ApoE $\varepsilon 4$ positive versus negative & & $-3.444(-7.411,0.522)$ & $2.89(1)$ & 0.089 \\
\hline ApoE $\varepsilon 4$ positive versus negative by week 26 & & $-1.716(-5.190,1.759)$ & $6.84(2)$ & 0.033 \\
\hline ApoE $\varepsilon 4$ positive versus negative by week 52 & & $-4.449(-7.831,-1.066)$ & & \\
\hline ApoE $\varepsilon 4$ unknown versus negative & & $3.455(-0.738,7.648)$ & $2.62(1)$ & 0.106 \\
\hline Baseline UPSIT & & $0.184(-0.034,0.402)$ & $2.76(1)$ & 0.097 \\
\hline$\triangle$ UPSIT & & $0.210(-0.148,0.567)$ & $1.32(1)$ & 0.25 \\
\hline
\end{tabular}

TS, testing statistic, which has a Chi-square distribution with degree of freedom (df) under the null hypothesis. ADAS-Cog, Alzheimer's Disease Assessment Scale-Cognitive (11 items, higher scores indicate worse cognitive performance); SRT, Selective Reminding Test 12 -item 6-trial version (higher scores indicate better cognitive performance); UPSIT, University of Pennsylvania Smell Identification test (40 items, range $0-40)$.

while the final model fit to 263 observations from 98 patients, because a patient with missing atropine challenge data dropped out after baseline.
The models in Table 3 show that ADAS-Cog scores decreased over time with a larger reduction from baseline to week 26 . ApoE $\varepsilon 4$ positive status was 
associated with higher ADAS-Cog scores, but the change of ADAS-Cog scores over time was unrelated to ApoE $\varepsilon 4$ positive status. Smokers had higher ADAS-Cog scores and more years of education than non-smokers. With education in the model, smoking status was no longer associated with ADAS-Cog and was excluded from the model. Controlling for the baseline variables, the variables of $\triangle$ UPSIT, indicator of week 26, and the term for interaction of $\triangle$ UPSIT by week 26 had significant coefficients while the indicator of week 52 and term for interaction of $\triangle$ UPSIT by week 52 did not, suggesting that the impact of $\triangle$ UPSIT was unstable and affected only the baseline ADAS-Cog and the change in ADAS-Cog from baseline to week 26. Particularly, higher $\triangle$ UPSIT was associated with lower ADAS-Cog at baseline $(\mathrm{B}=-0.04, p<0.001)$ and compared to those with negative $\triangle$ UPSIT, patients with positive $\Delta$ UPSIT showed less decline in ADAS-Cog from baseline to week 26.

For the two subjects with SRT data missing at 26 weeks, SRT total immediate recall scores were imputed by $19.7962-0.1776$ age +0.7965 (SRTtotal at week 8) based on data from 83 patients with good fitting $\left(R^{2}=0.7501\right)$. The initial linear model with repeated measures fit to 267 observations from 100 patients, and the final model fit to 266 observations from 99 patients because one patient who did not have $\triangle$ UPSIT dropped out after baseline.

SRT total immediate recall scores increased over time. Significant ApoE $\varepsilon 4$ by time interaction suggested that the status of ApoE $\varepsilon 4$ made a difference in the changes of SRT total immediate recall from baseline such that a significant increasing time trend occurred among the ApoE $\varepsilon 4$ negative patients with no significant changes among ApoE $\varepsilon 4$ positive patients. Controlling for education, smoking history was unrelated to SRT total immediate recall and was excluded from the final model. Controlling for the effect of ApoE $\varepsilon 4$ and other baseline variables, there was no effect of $\triangle$ UPSIT, nor $\triangle$ UPSIT by time interaction, on SRT total immediate recall (Table 3), indicating that response to atropine challenge was unrelated to SRT total immediate recall over time or the change in SRT total immediate recall from baseline to weeks 26 and 52. In contrast, SRT delayed recall did not change over time, with lower scores in older patients and ApoE $\varepsilon 4$ positives compared to negatives. Baseline UPSIT and $\triangle$ UPSIT, however, were unrelated to SRT delayed recall over time. Similar results were obtained with the secondary cognitive function measure, Everyday
Cognition (ECOG), which had higher scores in less educated females and ApoE $\varepsilon 4$ positives compared to negatives.

Improvement measured by CIBIC-plus scores at 26 or 52 weeks was positively associated with being ApoE $\varepsilon 4$ positive, baseline age and UPSIT score, while being unrelated to $\triangle$ UPSIT. There was no $\triangle$ UPSIT by time interaction on CIBIC-plus scores, indicating that $\triangle$ UPSIT was unrelated to CIBICplus over time. In exploratory analyses, there were no associations between $\triangle$ UPSIT and WAIS-R Digit Symbol scores over time ( 52 weeks).

FAQ scores (secondary measure) were unrelated to ApoE $\varepsilon 4$ status, baseline age, education and UPSIT score, while being lower in males than females $(p=0.014)$, and lower in past smokers than nonsmokers $(p<0.05)$, controlling for other baseline factors. There was no time trend in FAQ scores nor $\triangle$ UPSIT by time interaction on FAQ scores. A significant association of $\triangle$ UPSIT with FAQ scores ( $\mathrm{B}=-0.053, p=0.033$, in the model for square-root of FAQ controlling for baseline age, sex, education, smoking status, UPSIT score and time) indicated that $\triangle$ UPSIT had a non-linear association with lower FAQ over time.

\section{Change in UPSIT scores from 0 to 8 weeks}

Within-subject change in UPSIT scores from baseline to 8 weeks was not significantly different from zero, and was not associated with cognitive measures at weeks 26 and 52, nor with the change on the ADASCog or SRT total immediate recall from baseline to 26 weeks and from baseline to 52 weeks.

\section{Somatic side effects}

TESS scores, defined by the sum score of 26 items that cover possible physical side effects, decreased over time from 2.19 (SD 2.12) at baseline to 1.59 (SD 1.37) at 26 weeks and 1.47 (SD 1.80) at 52 weeks. There were no adverse effects of intranasal atropine reported or observed in any patient.

\section{Participants with improvement in CDR}

After excluding the ten participants with improvement in CDR, we did not find an interaction of $\triangle$ UPSIT by time nor effect of $\triangle$ UPSIT on ADAS$\mathrm{Cog}$, while the associations between ADAS-Cog and the other variables in Table 3 remained similar. For the other cognitive outcomes, the findings of no 
association with $\triangle$ UPSIT were unchanged while FAQ score became unrelated to $\triangle$ UPSIT. Odor identification test scores decreased with atropine challenge among the ten participants with CDR improvement ( $\triangle$ UPSIT mean $=-1.06, \mathrm{SD}=3.01$, $p<0.005)$, but not among the other 89 MCI patients ( $\triangle$ UPSIT mean $=0.30, \mathrm{SD}=8.04$ ). The difference between these groups, however, was not significant $(p=0.077)$.

Initial statistical power analysis for the sample size of 100 participants was based on calculated minimum detectable effect size with $80 \%$ power at significance level of alpha $=0.05$ for a two-sided test. The observed detectable correlation between outcome and predictor, $r=0.2757$ was moderate. Preliminary analysis of the final sample gave $r_{1}=0.2986$ for correlation between ADAS-Cog change over 26 weeks and the predictor (UPSIT change due to atropine nasal challenge) in 82 patients and $r_{2}=0.0983$ for correlation between ADAS-Cog change over 52 weeks and the predictor in 76 patients. The power of detecting $r_{1}$ and $r_{2}$ at alpha $=0.05$ was $78.7 \%$ and $12.5 \%$, respectively. Therefore, based on the results obtained, we did not have sufficient power to detect a small effect size that is needed for clinical relevance.

\section{DISCUSSION}

The decrease in odor identification test scores with atropine challenge was not associated consistently with improved cognitive performance during the 52-week open treatment trial with donepezil, with the sole significant finding of an increase in UPSIT with atropine nasal challenge being associated with decrease in ADAS-Cog scores at baseline and attenuated improvement over 26 weeks. The latter finding is not consistent with the associations postulated in the hypotheses. The association was not found in analyses excluding patients with improved CDR. Overall, the negative findings in this sample of 100 participants with MCI do not replicate other findings in much smaller samples [20]. The evaluation of olfactory function with atropine challenge, and the change in odor identification during the first 8 weeks, cannot be recommended for potential clinical use in predicting improvement in memory with CheI treatment. The presence of the apolipoprotein $\mathrm{E} \varepsilon 4$ allele affected some cognitive measures but did not alter the lack of association between change in odor identification test scores and cognitive outcomes.
Biomarkers for AD based on neuroimaging and cerebrospinal fluid (CSF) biomarkers were not obtained in this sample. One possible explanation for the negative findings is that several participants with MCI may not have had AD neuropathology and consequently would have been less likely to respond to CheI treatment. Cognitive scores generally improved to a small degree during the study and more patients showed improvement in CDR than worsening to dementia during the one-year study, lending support to this possibility. As noted, the statistical power was limited based on the detectable correlations that were observed.

There were other limitations to this study. While the associations of olfactory impairment on cognitive changes with open CheI treatment could be examined, the cognitive effects of cholinesterase inhibitors could not be assessed objectively in the absence of a placebo comparison group. Further, in the absence of a placebo comparison group, practice effects may have contributed to the results obtained, especially in the first 8 weeks [29]. The "Mecca" position may be uncomfortable for some participants, but it was used successfully without significant difficulty in another study that involved intranasal administration of atropine in older adults [19]. In our study, most patients did not experience significant difficulty with this procedure. We could not quantify the degree to which the atropine dose crossed the cribriform plate, but the significant decline in olfactory test performance in patients indirectly suggests that atropine did cross the cribriform plate.

Odor identification impairment is not specific to AD and occurs in Parkinson's disease, dementia due to Lewy bodies, schizophrenia, and other conditions [30]. In patients with Lewy body dementia, the association between improvement with CheI treatment and impaired odor identification has not been studied in a clinical trial [31]. In a small sample of patients with mild AD, a CSF Cholinergic index, defined as the ratio of CSF choline acetyltransferase to acetylcholinesterase, was shown to increase markedly in patients treated with the cholinesterase inhibitor galantamine compared to placebo [32]. Replication in larger samples is needed, with the caveat that there is limited clinical practical utility to the use of CSF assessment with lumbar puncture as a biological indicator of the therapeutic effect of cholinesterase inhibitors in $\mathrm{AD}$.

Prior efforts to establish efficacy of cholinesterase inhibitors in MCI were unsuccessful [27, 28], and this may be related to the heterogeneity in MCI with 
only some patients having AD neuropathology. In the future, targeting patients with MCI who have established AD biomarkers may be a more successful approach, both to evaluate the efficacy of treatments in MCI and to assess the utility of olfactory or other predictors of treatment response.

\section{ACKNOWLEDGMENTS}

This study was supported by a grant from the National Institute on Aging (R01AG041795).

Clinicaltrials.gov identifier: NCT01951118

Authors' disclosures available online (https:// www.j-alz.com/manuscript-disclosures/20-0021r1).

\section{SUPPLEMENTARY MATERIAL}

The supplementary material is available in the electronic version of this article: https://dx.doi. org/10.3233/JAD-200021.

\section{TRIAL REGISTRATION}

Olfactory Deficits and Donepezil Treatment in Cognitively Impaired Elderly. Clinicaltrials.gov identifier NCT01951118.

\section{REFERENCES}

[1] Ohm TG, Braak H (1987) Olfactory bulb changes in Alzheimer's disease. Acta Neuropathol 73, 365-369.

[2] Talamo BR, Rudel R, Kosik KS, Lee VM, Neff S, Adelman L, Kauer JS (1989) Pathological changes in olfactory neurons in patients with Alzheimer's disease. Nature 337, 736-739.

[3] Reyes PF, Deems DA, Suarez MG (1993) Olfactory-related changes in Alzheimer's disease: A quantitative neuropathologic study. Brain Res Bull 32, 1-5.

[4] Hyman BT, Arriagada PV, Van Hoesen GW (1991) Pathologic changes in the olfactory system in aging and Alzheimer's disease. Ann N Y Acad Sci 640, 14-19.

[5] Li W, Howard JD, Gottfried JA (2010) Disruption of odour quality coding in piriform cortex mediates olfactory deficits in Alzheimer's disease. Brain 133, 2714-2726.

[6] Devanand DP, Lee S, Manly J, Andrews H, Schupf N, Doty RL, Stern Y, Zahodne LB, Louis ED, Mayeux R (2015) Olfactory deficits predict cognitive decline and Alzheimer dementia in an urban community. Neurology 84, 182-189.

[7] Devanand DP, Lee S, Luchsinger JA, Andrews H, Goldberg T, Huey ED, Schupf N, Manly J, Stern Y, Kreisl WC, Mayeux R (2019) Intact global cognitive and olfactory ability predicts lack of transition to dementia. Alzheimers Dement 19, 35374-35379.

[8] Tabert MH, Liu X, Doty RL, Serby M, Zamora D, Pelton GH, Marder K, Albers MW, Stern Y, Devanand DP (2005) A 10-item smell identification scale related to risk for Alzheimer's disease. Ann Neurol 58, 155-160.
[9] Devanand DP, Liu X, Tabert MH, Pradhaban G, Cuasay K, Bell K, de Leon MJ, Doty RL, Stern Y, Pelton GH (2008) Combining early markers strongly predicts conversion from mild cognitive impairment to Alzheimer's disease. Biol Psychiatry 64, 871-879.

[10] Wilson RS, Schneider JA, Arnold SE, Tang Y, Boyle PA, Bennett DA (2007) Olfactory identification and incidence of mild cognitive impairment in older age. Arch Gen Psychiatry 64, 802-808.

[11] Davies P, Maloney AJ (1976) Selective loss of central cholinergic neurons in Alzheimer's disease. Lancet 2, 1403.

[12] Harvey JD, Heinbockel T (2018) Neuromodulation of synaptic transmission in the main olfactory bulb. Int J Environ Res Public Health 10, 15.

[13] Kasa P, Hlavati I, Dobo E, Wolff A, Joo F, Wolff JR (1995) Synaptic and non-synaptic cholinergic innervation of the various types of neurons in the main olfactory bulb of adult rat: Immunocytochemistry of choline acetyltransferase. Neuroscience 67, 667-677.

[14] Dolezal V, Kasparova J (2003) Beta-amyloid and cholinergic neurons. Neurochem Res 28, 499-506.

[15] Yamamoto Y, Shioda N, Han F, Moriguchi S, Fukunaga K (2010) Donepezil-induced neuroprotection of acetylcholinergic neurons in olfactory bulbectomized mice. Yakugaku Zasshi 130, 717-721.

[16] Smith RS, Araneda RC (2010) Cholinergic modulation of neuronal excitability in the accessory olfactory bulb. $\mathrm{J} \mathrm{Neu}$ rophysiol 104, 2963-2974.

[17] Velayudhan L, Lovestone S (2009) Smell identification test as a treatment response marker in patients with Alzheimer disease receiving donepezil. J Clin Psychopharmacol 29, 387-390.

[18] Pelton GH, Soleimani L, Roose SP, Tabert MH, Devanand DP (2016) Olfactory deficits predict cognitive improvement on donepezil in patients with depression and cognitive impairment: A randomized controlled pilot study. Alzheimer Dis Assoc Disord 30, 67-69.

[19] Schofield PW, Ebrahimi H, Jones AL, Bateman GA, Murray SR (2012) An olfactory 'stress test' may detect preclinical Alzheimer's disease. BMC Neurol 12, 24.

[20] Devanand DP, Lentz C, Chunga RE, Ciarleglio A, Scodes JM, Andrews H, Schofield PW, Stern Y, Huey ED, Bell K, Pelton GH (2017) Change in odor identification impairment is associated with improvement with cholinesterase inhibitor treatment in mild cognitive impairment. J Alzheimers Dis 4, 1525-1531.

[21] Grober E, Kawas C (1997) Learning and retention in preclinical and early Alzheimer's disease. Psychol Aging 12, 183-188.

[22] Folstein MF, Folstein SE, McHugh PR (1975) "Mini-mental state". A practical method for grading the cognitive state of patients for the clinician. J Psychiatr Res 12, 189-198.

[23] Hughes CP, Berg L, Danziger WL, Coben LA, Martin RL (1982) A new clinical scale for the staging of dementia. $\mathrm{Br}$ J Psychiatry 140, 566-572.

[24] Mohs RC, Rosen WG, Davis KL (1983) The Alzheimer's disease assessment scale: An instrument for assessing treatment efficacy. Psychopharmacol Bull 19, 448-450.

[25] Buschke H, Fuld PA (1974) Evaluating storage, retention, and retrieval in disordered memory and learning. Neurology 24, 1019-1025.

[26] Scheibe M, Bethge C, Witt M, Hummel T (2008) Intranasal administration of drugs. Arch Otolaryngol Head Neck Surg 134, 643-646. 
[27] Petersen RC, Thomas RG, Grundman M, Bennett D, Doody R, Ferris S, Galasko D, Jin S, Kaye J, Levey A, Pfeiffer E, Sano M, van Dyck CH, Thal LJ, Alzheimer's Disease Cooperative Study Group (2005) Vitamin E and donepezil for the treatment of mild cognitive impairment. $N$ Engl $J$ Med 352, 2379-2388.

[28] Salloway S, Ferris S, Kluger A, Goldman R, Griesing T, Kumar D, Richardson S, Donepezil 401 Study G (2004) Efficacy of donepezil in mild cognitive impairment: A randomized placebo-controlled trial. Neurology 63, 651-657.

[29] Duff K, Atkinson TJ, Suhrie KR, Dalley BC, Schaefer SY, Hammers DB (2017) Short term practice effects in mild cognitive impairment: Evaluating different methods of change. J Clin Exp Neuropsychol 4, 396-407.
[30] Masurkar AV, Devanand DP (2014) Olfactory dysfunction in the elderly: Basic circuitry and alterations with normal aging and Alzheimer's disease. Curr Geriatr Rep 3, 91-100.

[31] Stinton C, McKeith I, Taylor JP, Lafortune L, Mioshi E, Mak E, Cambridge V, Mason J, Thomas A, O’Brien JT (2015) Pharmacological management of Lewy body dementia: A systematic review and meta-analysis. Am J Psychiatry 172, 731-742.

[32] Karami A, Eriksdotter M, Kadir A, Almkvist O, Nordberg A, Darreh-Shori T (2019) CSF Cholinergic Index, a new biomeasure of treatment effect in patients with Alzheimer's disease. Front Mol Neurosci 12, 239. 